\title{
Design and Investigation of a Hybrid Optical Resonator for Medium Energy COIL
}

\author{
Karin M. Gruenewald and Juergen Handke \\ DLR, Institute of Technical Physics, Langer Grund, 74239 Hardthausen, Germany \\ Thomas Hall \\ DLR, Institute of Technical Physics, Pfaffenwaldring 38-40, 70569 Stuttgart, Germany \\ and \\ Frank Duschek \\ DLR, Institute of Technical Physics, Langer Grund, 74239 Hardthausen, Germany
}

\begin{abstract}
High brightness laser radiation of chemical oxygen iodine lasers (COIL) requires resonator types especially adapted to low gain medium and rectangular cavity geometry. For medium energy class lasers, those resonators suffer from small magnification numbers and therefore often imply sophisticated optical concepts. Selected solutions should not inhibit the demands of a field deployment of the laser system. Outside the laboratory additional nonoptical properties like compactness, ruggedness and ease of operation become important. Resonators that enable reliable operation under field conditions should be composed of a reasonable number of optical components and a straightforward architecture. Based on the amplification characteristics of a $10 \mathrm{~kW}$-class COIL, off-axis hybrid resonator configurations are designed and pre-evaluated theoretically. The most promising candidates are technically realized and applied to the DLR-COIL device. The resonators are tested for their alignment sensitivity and brightness parameters. Positive branch and negative branch hybrid resonators are discussed. Particularly, the negative branch hybrid resonator meets the above challenges. A further promising design approach, the multi-pass hybrid resonator, will be depicted within this paper.
\end{abstract}

\section{Introduction}

$\mathrm{D}$ IRECTED energy deployment of COIL ${ }^{1,2,3,4}$ demands excellent far field characteristics of the radiation. The resonator is no longer qualified by the extraction of high laser power only. A concurrent generation of high beam quality is required too. Stable resonator configurations can be employed with less technical effort for medium power level. But most of the straightforward concepts bear high Fresnel numbers in a range of 100. Accordingly, a $10 \mathrm{~kW}$-COIL beam extracted by a stable resonator is of high multimode and therefore of poor beam quality. High brightness operation suggests the application of unstable resonator geometries for a realization with reasonable technical effort. In literature, different resonator configurations $s^{5,6}$ are studied to enhance the COIL beam quality, and several sophisticated resonator concepts are successfully realized ${ }^{7,8,9}$. The basis of most considerations is the classical confocal unstable resonator.

In this paper hybrid resonators are presented that include off-axis modifications of the classical resonator type. Hybrid resonators combine a stable with an unstable resonator direction. The composition of two independent resonator directions allows a cylindrical mirror design that is perfectly adaptable to the rectangular COIL geometry. The stable resonator part is a simple Fabry-Perot configuration. The unstable part can be designed as negative branch or positive branch, depending on the curvature directions of the resonator mirrors.

Special emphasis is given to a reproducible and reliable resonator performance, simple resonator alignment, and the susceptibility to environmental disturbances. The resonator architectures are straightforward, consisting of a minimum of optical components and engineering effort. The goal is to facilitate the laser deployment under field conditions also by the simplicity of the resonator operation. 
Both hybrid resonator concepts are tested for their alignment and operating behavior, the extractable laser power and the achieved beam quality. The divergence of the extracted beam has to be close to the diffraction limit. The relation of measured divergence $\theta_{\text {meas }}$ to the optimum value $\theta_{\text {diff }}$ gives a first impression of the achieved beam quality BQ. This ratio is written as

$$
B Q=\frac{\theta_{\text {meas }}}{\theta_{\text {diff }}}
$$

The typical beam profile of COIL is top-hat. The diffraction limited divergence $\left(1 / \mathrm{e}^{2}\right)$ can be derived from the Fraunhofer diffraction at a rectangular aperture

$$
\theta_{\text {diff }}=\frac{0.7 \cdot \lambda}{2 \cdot d}
$$

The laser wavelength is given by $\lambda$, and $d$ is the beam width at the resonator aperture. The beam propagation ratio $\mathrm{M}^{2}$ is defined as

$$
M^{2}=\frac{(\theta \cdot \omega)_{\text {meas }}}{(\theta \cdot \omega)_{00}} \geq 1
$$

The beam propagation ratio relates the beam parameter product of the actual laser beam to the beam parameter product of an ideal Gaussian beam. The value of $\mathrm{M}^{2}$ should be closest to 1 . In the above equation, the beam divergence is given by $\theta$ and the waist radius of the beam is given by $\omega$. A correct application of the above equation is restricted to Gaussian and Gaussian-like laser radiation. The adaptation on top-hat COIL profile is only an approximation to receive first information on the laser brightness.

For a meaningful evaluation of laser output in any far field distance from the source, the average radiance ${ }^{10} \mathrm{R}$ is relating the laser power $\mathrm{P}$ to the corresponding emitting area multiplied by the solid angle. The according equation can be written for rectangular symmetry as

$$
R=\left[\frac{\pi}{4}\right]^{2} \cdot \frac{P}{M_{y}{ }^{2} \cdot M_{z}{ }^{2} \cdot \lambda^{2}}
$$

with the laser wavelength $\lambda$ and the beam propagation ratios in the directions $y, z$, perpendicular to the beam propagation direction $\mathrm{x}$. High laser output power combined with a beam propagation ratio close to 1 result in values for the average radiance as higher as lower the laser wavelength will be. The above definition of the average radiance is also valid for the description of the laser brightness. A criterion better adapted to COIL properties and COIL deployment might be the "power in the bucket". The power in the bucket determines the power within a defined solid angle in any distance to the laser source.

Supersonic COIL, as a fast cross-flow gas laser with an excellent scalability of laser power to highest values and a wavelength of $\lambda=1.315 \mu \mathrm{m}$, provides all characteristics for a high brightness laser system. The engineering task is given by the implementation of a resonator configuration that is capable of establishing the high beam quality while being optimally adapted to COIL gain medium.

\section{COIL Active Medium Properties and Demands}

The COIL cavity shows the typical geometry of cross-flow gas lasers. The re-pumping mechanism of iodine atoms by excited oxygen $\mathrm{O}_{2}\left({ }^{1} \Delta\right)$ expands the efficient laser active zone of DLR-COIL ${ }^{11}$ over $34 \mathrm{~mm}$ in flow direction. The dependence of laser power on the gain width is presented in figure 1 . The height of the flow channel is $25 \mathrm{~mm}$. The optimum resonator access to the COIL cavity therefore is rectangular. This resonator geometry guarantees a maximum exploitation of the active medium. Such a configuration is outlined in figure 2. In flow direction $\mathrm{z}$, the nozzle is followed by the laser cavity. In this sketch, the laser beam is extracted by a standard stable resonator

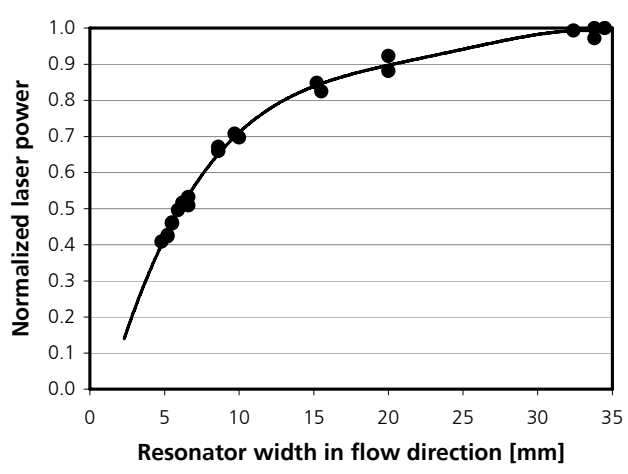

Fig. 1: Laser power versus resonator width 
in $\mathrm{x}$-direction. The resonator consists of a totally reflecting back mirror and a partially transmissive output mirror. Both circular mirrors are dimensioned to cover the large rectangular optical cross section.

In DLR-COIL, the gain medium is accelerated by a nozzle array to Mach 1.8 and a pressure of about 10 mbar and a temperature of 190 $\mathrm{K}$. The average small signal gain coefficient measured in center flow is about $1.1 \mathrm{~m}^{-1}$ and nearly constant along the flow axis. The experimental data are achieved by baseline operating conditions ${ }^{12,13}$. In the vertical direction, there is a broad central region of constant small signal gain with symmetric slopes in the boundary layers close to the upper and lower channel walls.

The power profile of figure 3 (bold marks) is measured for a variation of the output mirror reflectivity in a stable resonator. ${ }^{14}$ Figure 3 ad-

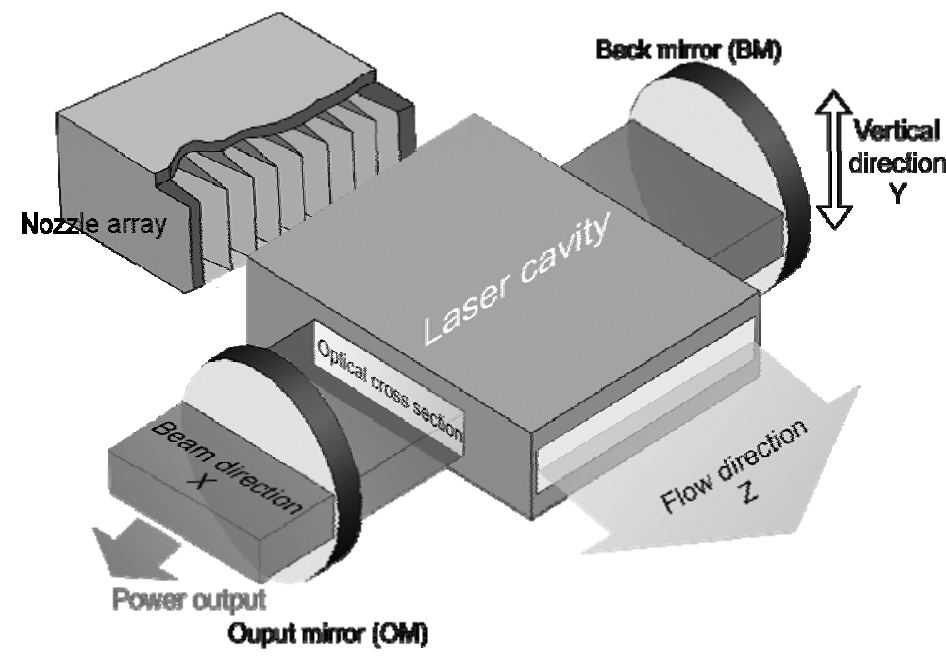

Fig. 2: Typical COIL geometry

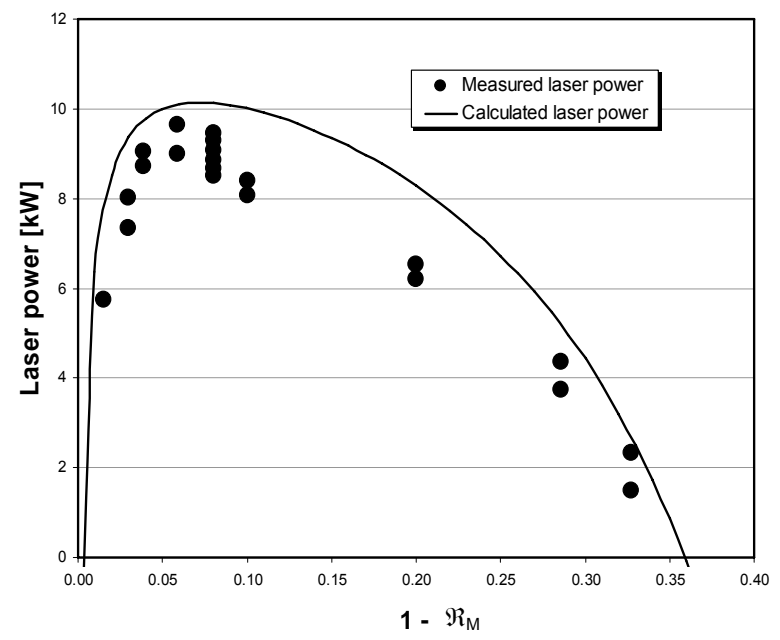

ditionally contents the corresponding Rigrod fit. The most fitting Rigrod curve yields a resonator with negligible losses. The average small signal gain coefficient derived from the Rigrod curve is about $1.3 \mathrm{~m}^{-1}$, little above the measured values. The relation between the total reflectivity $\mathfrak{R}_{\mathrm{M}}$ and the total loss coefficient $\mathrm{L}_{\mathrm{M}}$ given by

$$
\mathfrak{R}_{\mathrm{M}}+\mathrm{L}_{\mathrm{M}}=1 \text {. }
$$

The total loss coefficient includes the total outcoupling rate $\mathrm{O}_{\mathrm{M}}$.

The maximum laser power is achieved for an outcoupling rate of $6 \%$. The power steeply decreases for outcoupling rates below this value, and softly decreases above. About $90 \%$ of the maximum output power can be achieved for outcoupling rates within a range of $4 \%$ to $11 \%$. As a consequence of the above results, the optimum outcoupling derived from stable resonator is used for the determination of maximum allowed total loss in the following layout.

Fig. 3: COIL power profile with Rigrod fit

\section{Resonator Design}

High brightness performance of COIL suggests the application of unstable resonator geometries, though such resonators are reputed to be of complex maintenance, difficult to align and often critical in far field intensity distribution due to distinct diffraction structures. The diffraction loss within an unstable resonator is higher than the diffraction loss of a high Fresnel number, stable COIL resonator. Additional losses may occur by residual transmission of the nominally totally reflecting resonator mirrors.

Since the low gain COIL medium has to overcome the threshold for starting oscillation even of the enhanced resonator losses, the adaptation of an unstable resonator to a COIL of only $10 \mathrm{~kW}$-class is difficult. The resonator design as well as the predictions about its technical feasibility have to be performed carefully. An optimum design point can be found, when the theoretical layout is oriented on the amplification and outcoupling properties of the concerned laser system. With help of technical feasibility studies, possible shortcomings can be discovered already during the design stage and eliminated before manufacturing. 


\section{A. Theoretical Background}

The theory for the resonator layout is based on the integral equation of the Fresnel-Kirchhoff formulation of Huygens principle ${ }^{15}$

$$
E\left(y_{2}, z_{2}\right)=\iint_{A_{1}} K_{0}\left(y_{1}, z_{1} ; y_{2}, z_{2}\right) \cdot N_{1}\left(y_{1}, z_{1}\right) \cdot N_{2}\left(y_{2}, z_{2}\right) \cdot E\left(y_{1}, z_{1}\right) \cdot d y_{1} d z_{1}
$$

and is applied to passive resonators. ${ }^{16,17}$ The equation describes the development of field $\mathrm{E}\left(\mathrm{y}_{2}, \mathrm{z}_{2}\right)$ on mirror area $\mathrm{A}_{2}$. Mirror area $A_{2}$ is set-up in the layer $\left[\mathrm{y}_{2}, \mathrm{z}_{2}\right]$ perpendicular to the beam propagation direction $\mathrm{x}$. The field $\mathrm{E}\left(\mathrm{y}_{2}, \mathrm{z}_{2}\right)$ depends on the field $E\left(y_{1}, z_{1}\right)$ on mirror area $A_{1}$ in the layer $\left[y_{1}, z_{1}\right] . K_{0}$ is the kernel of the integral equation for plane mirrors. The correction according to the particular mirror curvatures is given by $\mathrm{N}_{1}$ and $\mathrm{N}_{2}$, respectively. Provided the kernel is separable in the two directions, the field is assumed to be written as $\mathrm{E}(\mathrm{y}, \mathrm{z})=\mathrm{E}(\mathrm{y}) \cdot \mathrm{E}(\mathrm{z})$ and the calculation can be reduced to a one-dimensional problem by solving two non-interacting equations for $E\left(\mathrm{y}_{2}\right)$ and $E\left(z_{2}\right)$. The numerical integration is performed by the Fox-Li-method ${ }^{18}$.

The computation yields the final value for the magnification and the complete resonator geometry. Moreover the related intensity profiles in the near field and far field are calculated. Additional studies on the design sensitivity and the alignment sensitivity can follow. A further result of the calculation is the total loss. The total loss is indicated as total coupling loss and consists of two different parts: the loss due to the power output (output coupling) and the loss due to diffraction effects (diffraction loss). Further mirror losses are neglected.

\section{B. Interaction of Theory and Experiment}

Theory and experimental results are brought together by initial and boundary conditions for the computation. Experimental data like the efficient amplification area in the cavity give a first hint for the mirror dimensions, and the range of efficient outcoupling indicates the maximum allowed total loss of the unstable resonator.

The overall mirror dimensions are based on this information and the resonator length follows from a reasonable estimation.

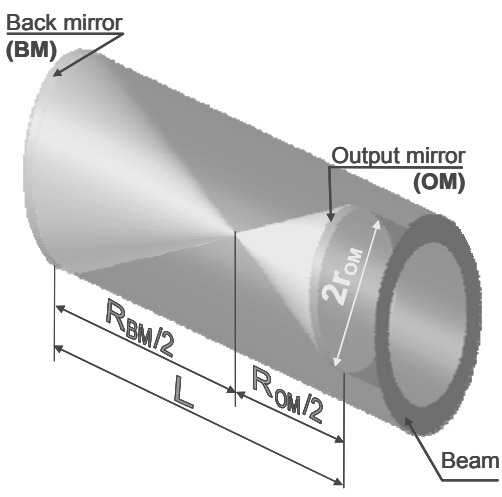

Fig. 4: Classical confocal unstable resonator The sketch of a classical confocal unstable resonator is given in figure 4. The geometrical output coupling, A, (no diffraction effects included) is given by

$$
A=1-\frac{1}{M^{2}}
$$

The above equation is based on the assumption of a plane wavefront leaving the resonator. A first value of the magnification, $\mathrm{M}$, can be obtained, if the geometrical output coupling is assumed to correspond to the experimental total coupling loss. According to the definition of the magnification

$$
M=\frac{R_{B M}}{R_{O M}}
$$

the radii of curvature of the resonator mirrors can be defined. $R_{\mathrm{BM}}$ is the radius of curvature of the back mirror (BM) and $R_{\mathrm{OM}}$ is the radius of curvature of the output mirror $(\mathrm{OM})$. In an iterative process, the magnification number is fitted until the calculated total coupling loss complies with the coupling range of the Rigrod curve. For maximum outcoupling, the value of the output coupling should be close to the total coupling loss.

\section{Theoretical Studies}

The resulting resonator configuration is put through a feasibility study, before any engineering effort is invested. The feasibility study checks the design sensitivity and the alignment sensitivity of the system. The effects of small deviations from the required radii of curvatures on resonator losses as well as the effects of mirror tilt are calculated for each proposed resonator configuration. The dependence of resonator losses on the radii of curvatures results in the necessary degree of precision for the mirror specification and in reliable information on the acceptable manufac- 
turing tolerances. Beside of the basic information on the feasibility, the knowledge of the design sensitivity helps to estimate the resonator hardware costs. The alignment sensitivity can be predicted by relating the resonator losses to the mirror tilt. Together with the calculated intensity distributions, the design sensitivity and the alignment sensitivity support the decision on the technical realization of any proposed resonator concept. ${ }^{19}$

\section{Hybrid Resonator Performance and Perspectives}

\section{A. Resonator Configuration}

The above addressed difficulties due to the small amplification and the rectangular geometry are illustrated in figure 5, where a classical unstable resonator is adapted to the COIL cavity. For this conventional resonator, the beam is

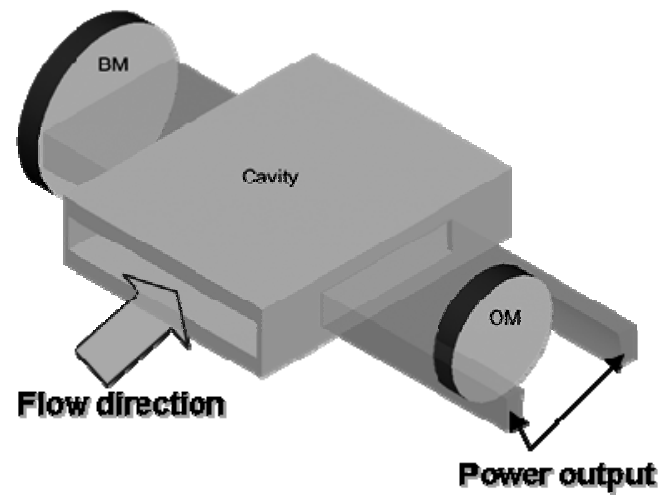

Fig. 5: Classical unstable resonator adapted to COIL geometry coupled out of two separate apertures. Each aperture is very narrow as a direct consequence of the small magnification. Consequently, a resulting far field distribution would be predominated by diffraction structures, and the beam

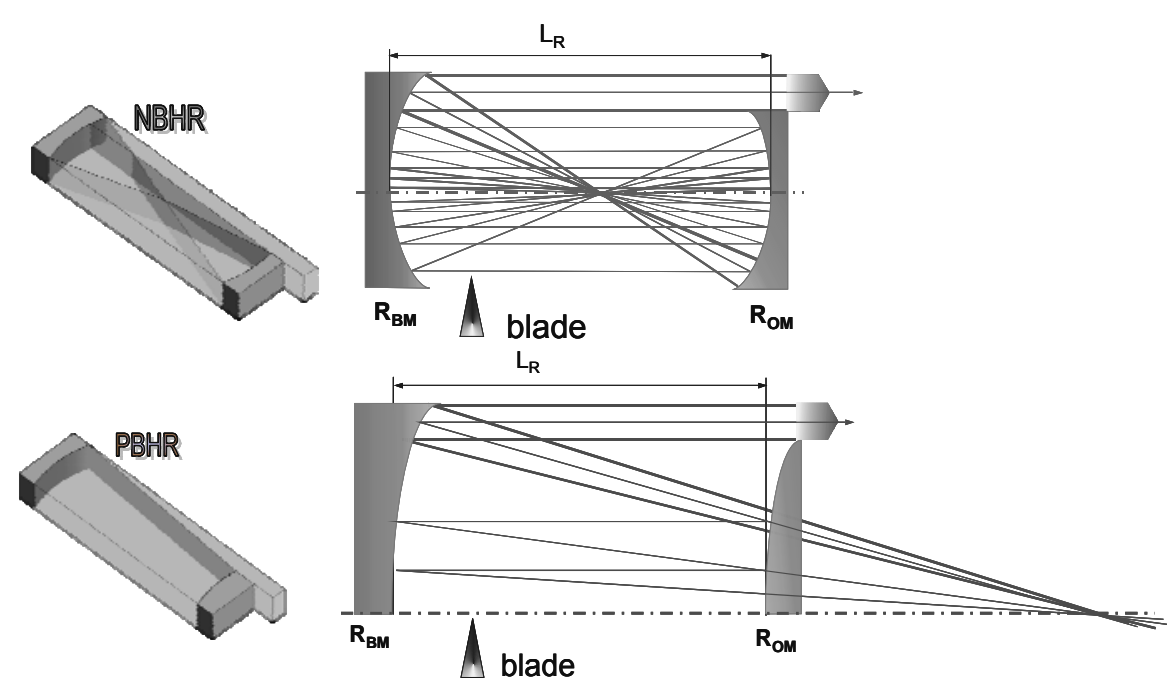
quality would be rather poor. Furthermore, maximum power output requires an optimum exploitation of the gain medium. Therefore, the resonator has to be applied to the complete area of optical access.

Specific modifications of the standard resonator have to be performed to meet both conditions, maximum power output (e. g. by filling factor) and optimum beam quality (e. g. by well-balanced aperture).

The rectangular geometry of COIL optical access is optimally adapted to the cy-

Fig. 6: Well adapted resonator configuration for COIL NBHR: Negative branch hybrid resonator PBHR: Positive branch hybrid resonator lindrical mirrors of hybrid resonators. For DLR-COIL, the resonator directions are

oriented as follows: The unstable resonator direction passes along the gas flow and the stable resonator part is implemented perpendicularly to the gas flow in vertical direction.

Figure 6 shows two types of hybrid resonators that are indicated as NBHR (negative branch confocal hybrid resonator) and PBHR (positive branch confocal hybrid resonator). For both, the stable part is realized by a FabryPerot resonator (FPR). The indications "negative branch" and "positive branch" refer to the corresponding quadrants of the stability diagram ${ }^{20}$. In both sketches of figure 6 the optical axis is symbolized by the dash-dotted lines. The radiation field inside the NBHR generates a focal line. The optical axis of the PBHR is located at the mirror edges and the radiation is continuously putting an increasing distance to the axis. The blade is used to protect the cavity from scattered or misaligned radiation

The resonator height and the allowed magnification determine the aspect ratio of the output beam, i.e. the ratio of the beam dimensions in stable and unstable direction. In unstable direction the off-axis modification enables power extraction from a single aperture. This single aperture enables a more favourable ratio of enclosed area to circumference than the outcoupling from classical unstable resonator. 


\section{B. Feasibility Studies -Theoretical results}

The design sensitivity of a particular NBHR configuration is shown in figure 7. The contour plots show the output coupling and the diffraction losses depending on the radii of curvatures for back mirror and output mirror, according to a desired resonator length of $3 \mathrm{~m}$.
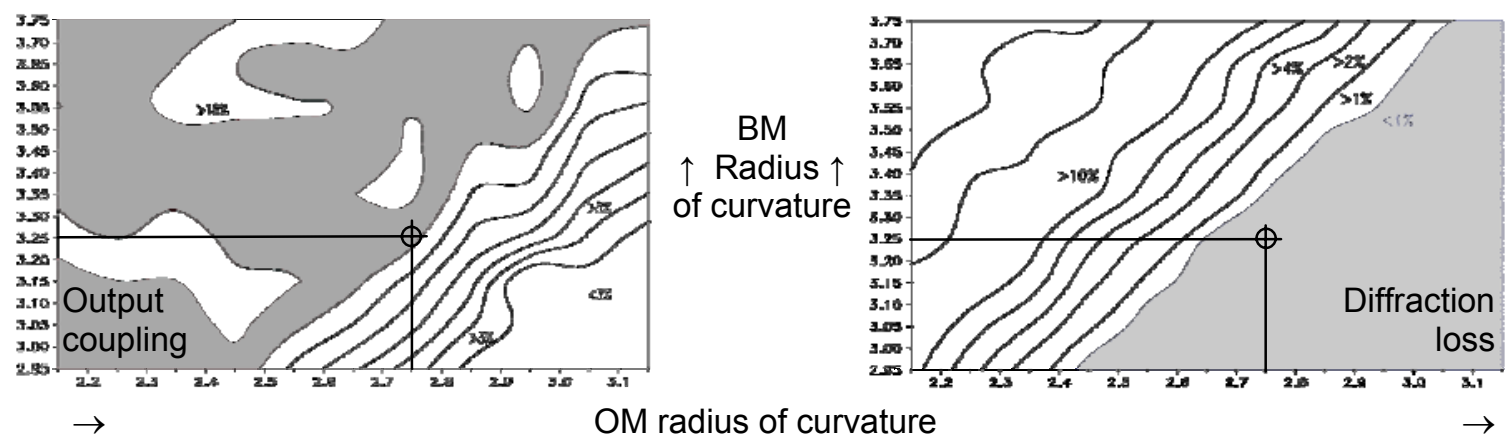

Fig. 7: NBHR design sensitivity for a nominal resonator length of $3 \mathbf{~ m}$; actual resonator length adapted to the actual radii combination, according to the deviations from the nominal radii

The layout data of the radii are marked in both diagrams by the intersection of the straight lines. For the specific layout curvatures, a surrounding area for several centimeters of radii is encircled. Within this area, no significant change of losses will occur. This validity is based on the assumption, that the nominal resonator length is adapted to the true mirror curvatures. This marked region complies with allowable manufacturing tolerances, which can be covered by the skills of industrial manufacturing.

The alignment sensitivity for such a resonator is studied in stable and unstable direction separately. For both directions, the effects of mirror tilts on power output and total coupling loss are investigated. The influence on the diffraction loss can be derived from the difference between the total coupling loss and the output coupling. The tilt angles in unstable direction, $\beta$ for back mirror and $\alpha$ for output mirror, are specified by the right hand side sketch of
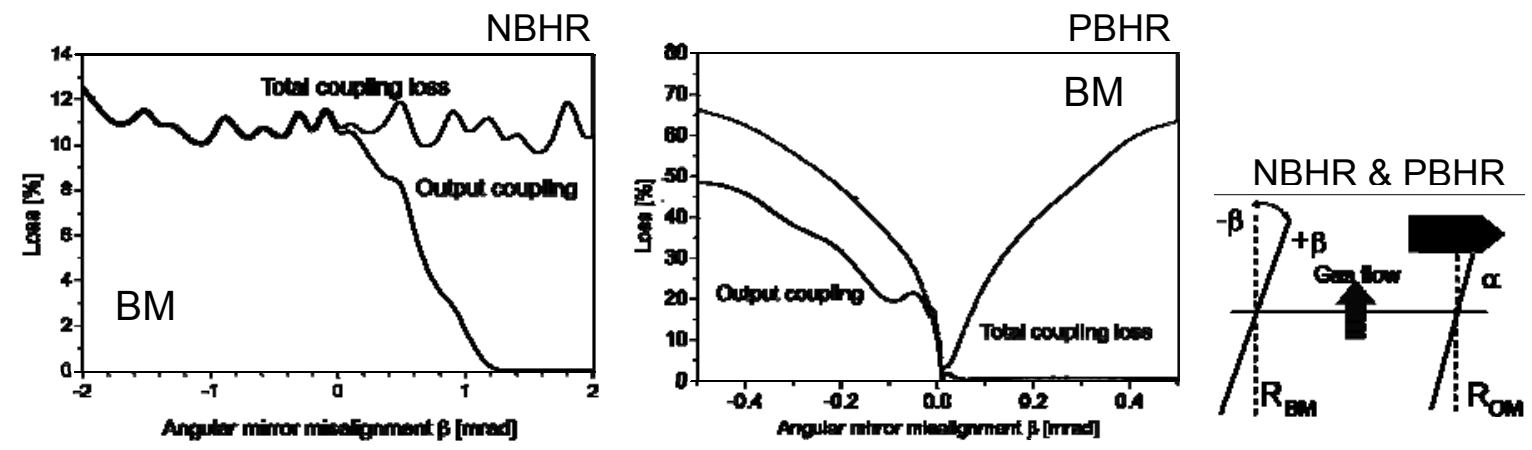

Fig. 8: Comparison of NBHR- and PBHR-alignment sensitivity for back mirror tilt $(\beta)$ in unstable direction

figure 8. A comparison of NBHR and PBHR alignment sensitivities can be deduced from the figures 8 and 9.

In figure 8, the loss reactions on back mirror tilts for NBHR and PBHR are compared. A tilt of NBHR back mirror in flow direction $(-\beta)$ allows a large tilt angle with no remarkable change in loss. When the same mirror is tilted against the flow direction $(+\beta)$, the resonator axis moves towards the nozzle array. In this case, the extractable power strongly decreases, while the diffraction loss increases with nearly the same gradient. As a result of both, the total coupling loss keeps unchanged. A symmetric alignment accuracy of $\pm 200 \mu \mathrm{rad}$ can be derived from these considerations for NBHR. The response of the PBHR losses is by at least one order of magnitude larger than those of NBHR. A tilt of the mirror against the flow axis $(+\beta)$ directly pushes the optical axis out of the resonator area as a consequence of the off-axis configuration. Therefore, the alignment accuracy for PBHR demands a smaller angular range of only $\pm 20 \mu \mathrm{rad}$.

A tilt of the NBHR output mirror shows a more symmetric response of the losses, as presented in figure 9. Only slight variations will occur within an angle of about $\pm 500 \mu \mathrm{rad}$. PBHR again reacts very sensitive on the mirror tilt, 
as to be seen in figure 9. The maximum allowed tilt angle of PBHR is the same as for the back mirror. Additionally, figure 9 shows the total coupling loss in Fabry-Perot direction that is identical for both resonator types. For the stable direction, the aligning accuracy is demanding for PBHR as well as for NBHR. The tilt should be smaller than \pm 20urad.
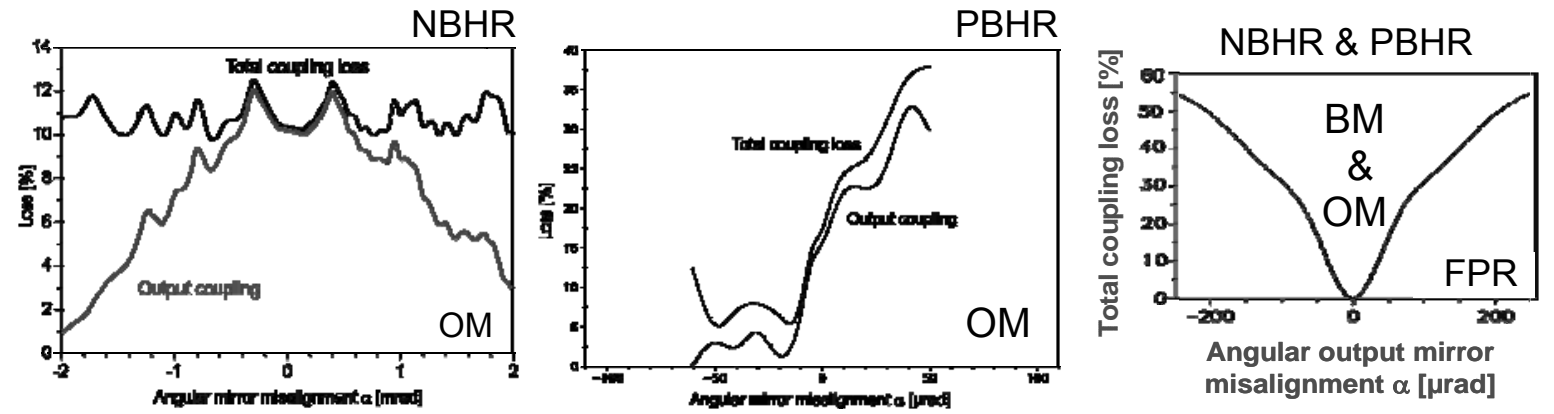

Fig. 9: Comparison of NBHR- and PBHR-alignment sensitivity for output mirror tilt $(\alpha)$ in unstable direction and for both resonators and mirrors in stable direction

As a result of the loss responses, a more promising alignment probability can be derived for the NBHR. The alignment in stable direction, however, turns out to be difficult even for NBHR. With a maximum allowed tilt angle of only a few ten $\mu$ rads, the required accuracy is by one order of magnitude higher then in unstable direction. For PBHR, the necessary aligning accuracies of both directions are of the same level.

\section{Experimental Results}

Both types of resonators have been manufactured and tested for comparable magnification, 1.11 and 1.13 , and output coupling of $9.4 \%$ for NBHR and $9.6 \%$ for PBHR, respectively. The nominal resonator length is $2 \mathrm{~m}$. With the results of the theoretical studies, automatic alignment procedures have been elaborated and cross checked by the output power response of the resonator. Figure 10 shows the effect of angular back mirror tilt on the extractable laser power. The experimental results are in very good agreement with the theoretical predictions of figure 8 and figure 9, respectively. The decrease of laser power directly corresponds to the reduced output coupling.
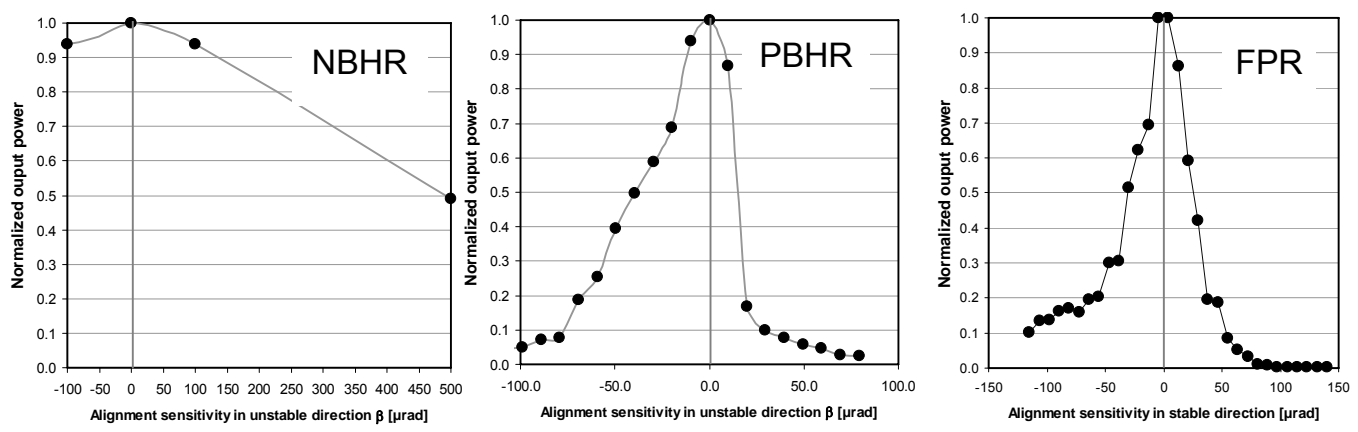

Fig. 10: Measured laser power dependent on resonator alignment of the unstable direction of NBHR and PBHR and the stable direction of both (FPR: Fabry Perot $\underline{\text { Resonator) }}$

As predicted, the NBHR is by far easier to align than the PBHR. The alignment procedure is made up of three steps: After a pre-alignment by a HeNe laser, a first rough scan of the tilt angles is performed, as outlined in the sketch on the left side of figure 11 . This step is followed by a subsequent fine alignment. The rough scan determines the optimum angular mirror position in unstable direction given by the maximum power peak. Additionally it gives a first hint for the optimum position in stable direction. The following fine alignment is performed in stable direction. The mirror is automatically tilted until the maximum power is achieved. The definite alignment of the resonator is achieved by this last step. The resonator works stable and reliable for all following operation periods as long as no mechanical modifications are carried out. 

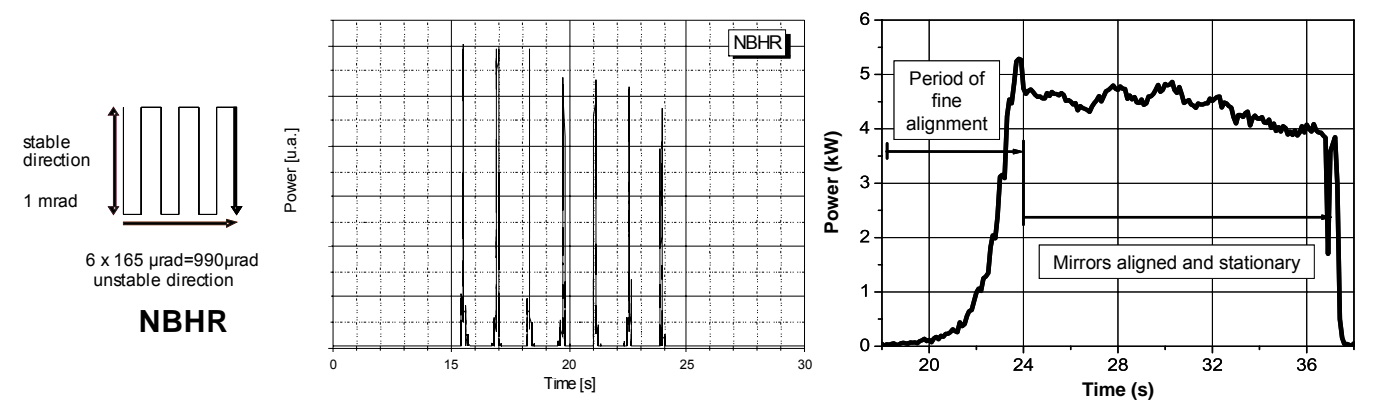

Fig. 11: Alignment procedure and power response on output mirror tilt of NBHR

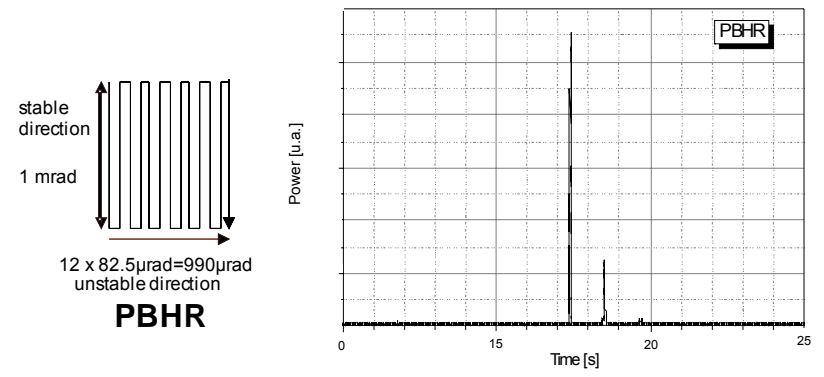

Fig. 12: Alignment procedure and power response on output mirror tilt of PBHR
The described alignment method is more difficult to apply on PBHR. According to the theoretical prediction, the necessary alignment accuracies for the both PBHR directions are of the same order of magnitude. Figure 12 shows that the laser oscillation is limited to a much smaller tilt angle in unstable direction. Consequently a narrower scan is performed to localize the optimum in unstable direction. From the power response in figure 12 directly follows that an even further fine tuning would be necessary to identify the correct optimum.

The power coupled out of NBHR achieved about $7 \mathrm{~kW},{ }^{21}$ see figure 13 . The theoretically achievable laser power for the chosen output coupling and the calculated diffraction losses is about $8 \mathrm{~kW}$. The maximum power output of PBHR is difficult to determine, due to the alignment efforts. $5 \mathrm{~kW}$ of laser power could be easily detected with reasonable alignment effort. A further enhancement of laser power assumes an even more precise alignment.
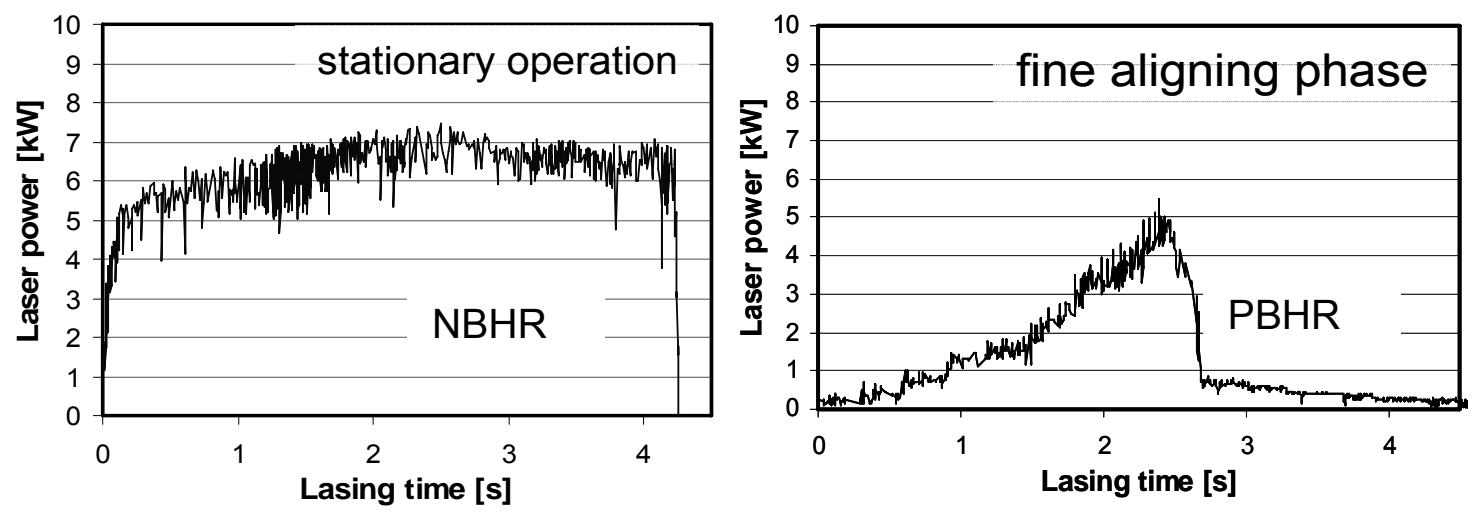

Fig. 13: Laser power coupled out of NBHR and PBHR

An inherent problem of NBHR is attached to its internal focal line that may induce a plasma breakdown or at least some distortion within the cavity. In a first configuration, the NBHR is implemented in a way that the focal line is positioned far outside the laser gain medium. Further tests were performed with the focal line attached closer to the active medium until the line was positioned just in the middle of the gain medium. Independently of the position of focal line, a distortion of the active medium was not detected during the test series and beyond, the resonator performance was not affected by the position of the focal line. The laser intensity distribution is measured by a CCDcamera (SPIRICON, COHU 4812) that is well suited for the $1.3 \mu \mathrm{m}$ wavelength. The far field intensity distribution 

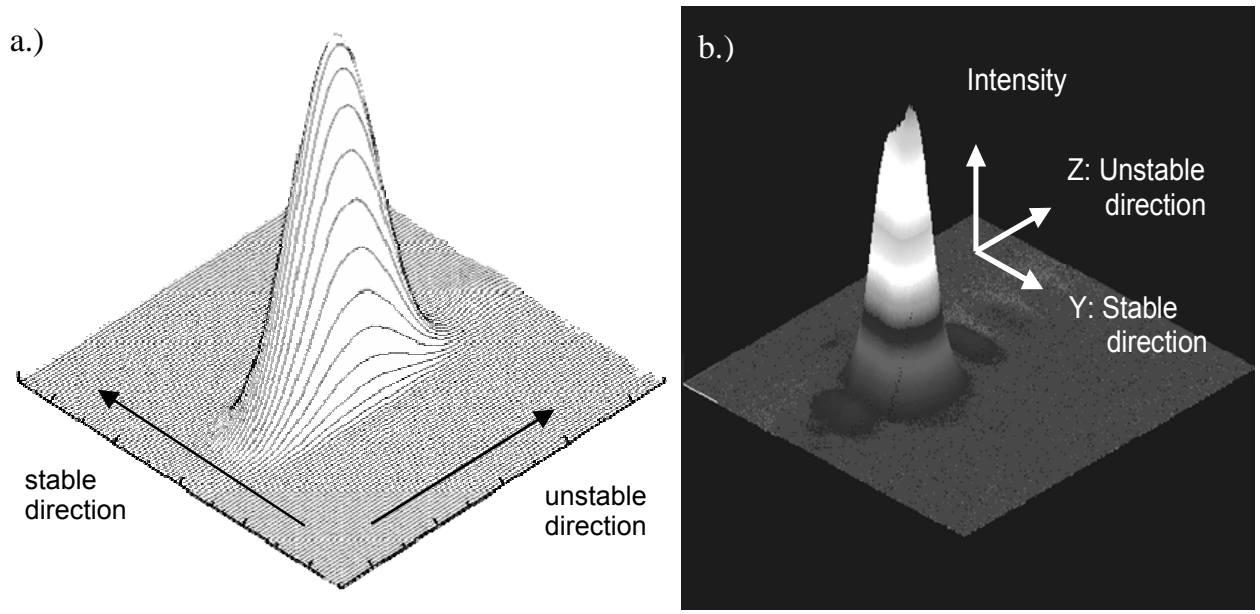

Fig. 14: Far field distribution of NBHR: a.) Calculated two-dimensional profile,

b.) Measured intensity profile

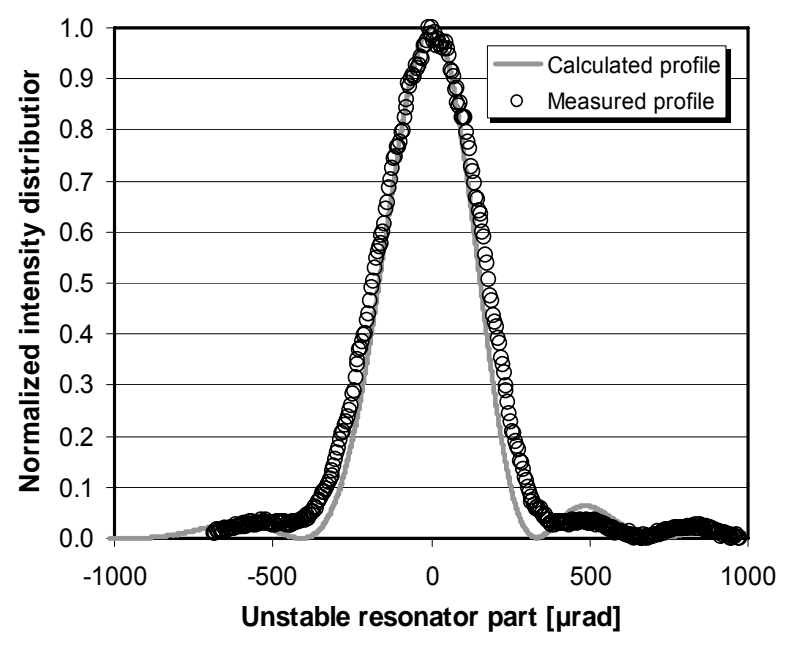

of NBHR is presented in figure 14. The beam quality, as defined in the introduction, is $\mathrm{BQ}=1.4$ in unstable direction and $\mathrm{BQ}=7.9$ in stable direction, due to the large Fresnel number of $\mathrm{N}_{\mathrm{F}}=60$. The average radiance can be approached to a value of about $\mathrm{R}=1 \cdot 10^{14} \mathrm{Wm}^{-2}$ ster $^{-1}$.

The predicted and the measured profiles for the intensity distribution are found to be in excellent agreement, as shown in figure 15 for the unstable

Fig. 15: Comparison of calculated and measured NBHR profile in unstable direction

direction of NBHR.

A second test series with the PBHR configuration provides similar, but slightly weaker beam qualities compared to the NBHR series. The measured far field intensity distribution of PBHR is presented in figure 16 . The reason for the weaker results of PBHR can be attributed to the higher aligning demands. When taking also into consideration that the pointing stability of NBHR exceeds the values of PBHR ${ }^{22}$ the NBHR is in every respect the preferable resonator concept.

\section{Multi-Pass Hybrid Resonator}

While NBHR achieves good beam quality in unstable direction, the beam quality in stable direction has to be improved. In stable direction, a simple reduction of the Fresnel number should enhance this value. Experiments with NBHR of reduced channel height lead to a laser emission that is closer to the diffraction limit.

Figure 17 shows the dependence of the measured divergence on the NBHR resonator height. The beam quality in stable direction decreases according to the decrease of the Fresnel number. ${ }^{23}$ Since the unstable direction is also af- 


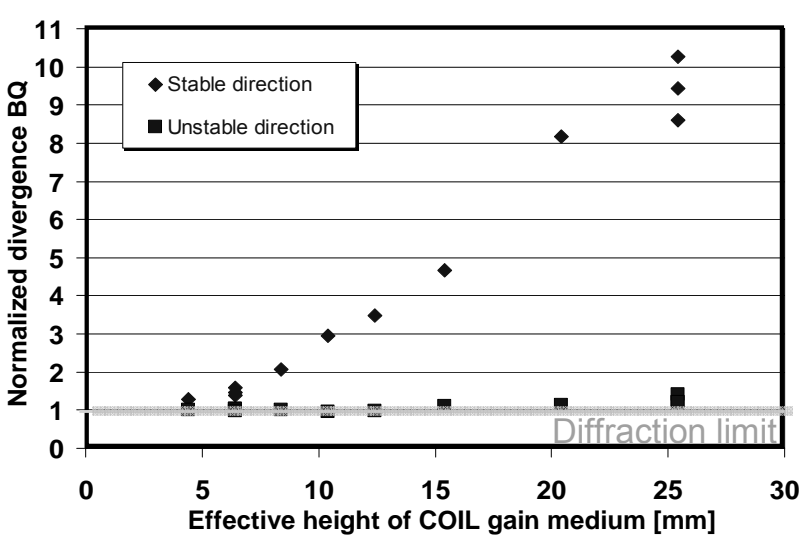

Fig. 17: Far field dependence on mirror dimensions fected by the resonator height, the stable and the unstable direction seem not to be completely separated. This fact may follow from an imperfection of the mirror set-up or of inaccuracies in mirror manufacturing.

A reduction of resonator height also yields an unwanted decrease in laser power according to the reduced exploitation of active medium. This can

be avoided by folding of the resonator in stable direction. This way, the complete medium can be exploited and the Fresnel number is further on reduced by increasing the resonator length. Figure 18 shows a sketch of a double-pass NBHR. The achievable power will depend on the realized filling factor. Based on the single-pass measurements, the average radiance of such a configuration is expected in the range of $\mathrm{R}=5 \cdot 10^{14} \mathrm{Wm}^{-}$ ${ }^{2}$ ster $^{-1}$.

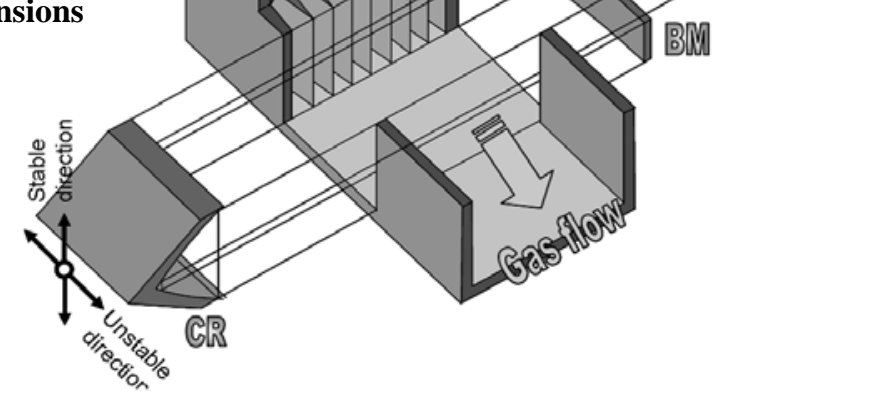

Fig. 18: Double-pass NBHR

\section{Conclusion}

Hybrid resonators reliably fulfill the request for high brightness COIL operation of a $10 \mathrm{~kW}$ class COIL. The cylindrical mirrors are perfectly adaptable to COIL geometry. Minor disadvantages of hybrid resonators originate in the high initial costs of the cylindrical mirrors as well as by the inherent stable resonator part.

Among the hybrid resonators, the NBHR is given priority due to the simpler alignment. About $70 \%$ of maximum laser power is coupled out of NBHR. The laser radiation is about 1.4 times diffraction limited in unstable direction. In stable direction, the radiation is only 7.9 times diffraction limited due to the high Fresnel number. The average radiance can be derived to $1 \cdot 10^{14} \mathrm{Wm}^{-2} \mathrm{ster}^{-1}$. Beam degradation due to the focal line of NBHR was not observed.

The experimental results of both types of hybrid resonators are in excellent agreement with the theoretical predictions. This is valid for the resonator design and alignment sensitivities as well as for the resonator performance.

A further improvement of hybrid resonator performance will be achieved by multi-pass geometry. The resonator has to be folded in the stable direction. A simple double-pass system will enhance the combined (of both resonator directions) beam propagation ratio to a value better than $\mathrm{M}^{2}=2$. An enhancement of brightness will also depend on the extractable power and therefore on the filling factor actually achieved in the folding direction. Values of brightness in the range of $5 \cdot 10^{14} \mathrm{Wm}^{-2} \mathrm{ster}^{-1}$ are expected for double-pass NBHR.

\section{Acknowledgments}

The authors like to express their gratitude to Dr. Gerhard Spindler, whose good ideas, experience, and essential contributions to the discussions were a great support during the investigations and made a valuable contribution to this paper. 


\section{References}

1. W.L.Bohn, "Medium Energy Laser (MEL)", US-German MOU-Meeting, Albuquerque, NM, USA, 2003.

2. W.L. Bohn, "German COIL efforts: status and perspectives", Photonics West, San Jose (California USA) (edited by Heaven, Steven J. Davies Michael C.), Gas and Chemical Lasers and Intense Beam Applications III, Proc. SPIE, Vol. 4631, 53 - 59, 2002.

3. A.S. Boreysho, "High-power mobile chemical lasers", Quant. Electr., 35(5), 393 - 406, NY, 2005.

4. A. Boreysho, "Integration of COIL in High-Power Laser System", XV Int. Symp. on Gas Flow, Chemical Lasers, and High-Power Lasers, (edited by J. Kodymová, Praque 2004), Proc. SPIE, 5777, 16-31, Bellingham, WA, 2005.

5. S.L. Druzhinen, A.V. Savin, S.Yu. Strakhof, "Unstable multi-pass resonator for ten-kW-class nitrogen-based supersonic COIL" XV Int. Symp. on Gas Flow, Chemical Lasers, and High-Power Lasers, (edited by J. Kodymová, Praque 2004), Proc. SPIE, 5777, 135-141, Bellingham, WA, 2005.

6. A.H. Paxton, W. P. Latham, Jr., "Unstable resonators with $90^{\circ}$ beam rotation", Appl. Opt., 25(17), 2939-2946, 1986.

7. S. Holswade, R. Riviere, C.A. Huguley, Ch. M. Clayton, G.C. Dente, "Experimental evaluation of an unstable ring resonator with 90 beam rotation: HiQ experimental results", Appl, Opt., 27(21), 4396-4406, 1988.

8. D. Yu, F. Sang, Y. Jin, Y. Sun, "Output beam analysis of an unstable resonator with a large Fresnel number for a chemical oxygen iodine laser", Journal of Optical Engineering, Vol. 41, (10), 2668-2674, 2002.

9. Y. Jin, B. Yang, F. Sang, D. Zhou, L. Duo, Q. Zhuang, " Experimental investigation of an unstable ring resonator with 90-deg beam rotation for a chemical oxygen laser", Appl. Opt., 38(15), 3249-3252, 1999.

10. A.V. Kudryashov, H. Weber (Editors) "Laser Resonators, Novel Design and Development", SPIE Press, Bellingham, 1999.

11. L.v. Entress-Fürsteneck, J. Handke, K. Grünewald, "Supersonic COIL operation at DLR, Germany", $11^{\text {th }}$ GCL/HPL, SPIE, 3092, 553 - 556, Edinburgh, 1996.

12. K.M.Grünewald, J. Handke, W.O. Schall, L. v.Entress-Fürsteneck, "Effects of the Gas Mixing on COIL Performance", $12^{\text {th }}$ GCL/HPL, SPIE, 3574, pp. 315-320, St. Petersburg, 1998.

13. K.M. Grünewald, J. Handke, F. Duschek, "Small signal gain and temperature profiles in supersonic COIL", $13^{\text {th }}$ GCL/HPL, SPIE, 4184, pp. 75 - 78, Florence, 2000.

14. J. Handke, K.M. Grünewald, F. Duschek, "Comparative studies on small signal gain and output power for COIL systems", 13 ${ }^{\text {th }}$ GCL/HPL, SPIE, 4184, 45 - 48, Florence, 2000.

15. J.W.Goodman, "Introduction to Fourier Optics", McGraw-Hill, 1968.

16. Th. Hall, F. Duschek, K.M. Grünewald, J. Handke, W.O. Schall, "Numerical Studies on Hybrid Resonators for COIL". XV Int. Symp. on Gas Flow, Chemical Lasers, and High-Power Lasers, (edited by J. Kodymová, Praque 2004), Proc. SPIE, 5777, 131 - 134, Bellingham, WA, 2005.

17. Th. Hall, "Numerical studies on hybrid resonators for a medium-sized chemical oxygen iodine laser", Opt. Eng. 44(11), 114201, 2005.

18. A.G. Fox, T. Li, "Resonant Modes in a Maser Interferometer", Bell Syst.Techn. Journ., 40, 453 ff, 1961.

19. J. Handke, F. Duschek, K.M. Grünewald, T. Hall, W.O. Schall, "Near diffraction limited high power COIL emission", XV Int. Symp. on Gas Flow, Chemical Lasers, and High-Power Lasers, (edited by J. Kodymová, Praque 2004), Proc. SPIE, 5777, 127 - 130, Bellingham, WA, 2005.

20. H. Kogelnik, T. Li, "Laser Beams and Resonators", Journal of Applied Optics, 5(10), pp. 1550-1567, 1966.

21. J. Handke, W. O. Schall, T. Hall, F. Duschek, and K. M. Grünewald, "Chemical oxygen-iodine laser power generation with an off-axis hybrid resonator," Appl. Opt. 45, 3831-3838, 2006.

22. K. Yasui, Y. Takenaka, "Advantages of negative-branch compared with positive-branch one-dimensional unstable resonators", Appl. Opt., 40(21), 3547-3551, 2001.

23. Karin M. Gruenewald, "Resonators for High Brightness COIL", $12^{\text {th }}$ Conference on Laser Optics, June 26-30, to be published in SPIE, St. Petersburg, 2006. 